\title{
Analysis of factors affecting the operation of the communication system with non- orthogonal multiple access
}

\author{
Eugeniy Rogozhnikov ${ }^{1, *}$, Yakov Kryukov ${ }^{1}$, Dmitriy Pokamestov ${ }^{1}$, Edgar Dmitriev ${ }^{1}$, \\ and Renat Abenov ${ }^{1}$ \\ ${ }^{1}$ TUSUR, Department of Telecommunications and Basic Principles of Radio Engineering, \\ Tomsk, Russian Federation
}

\begin{abstract}
NOMA is a group of non-orthogonal multiple access methods [1]. In this article, we talk about the Power Domain (Division) NOMA method. It is a non-orthogonal multiple access method with channel separation by power [2]. NOMA compaction uses controlled inter-channel interference, which is removed in the receiver when channel separation using special algorithms. PD-NOMA can be used in conjunction with various types of modulation, including OFDM.
\end{abstract}

\section{Introduction}

The advantage of PD-NOMA technology allows you to increase the adaptability in the allocation of communication resources by taking into account the additional domain (power) for the distribution of channels. This increases the spectral efficiency of a multichannel communication system compared to orthogonal methods. PD-NOMA also allows you to increase the number of user channels per unit of time-frequency resource.

The PD-NOMA method has a few problems.

1) Demodulation errors in the lower layer channels cause additional demodulation errors in the upper layer channel.

2) Power calculation problem. Signal-code constructions impose their limitations on the range of permissible power relations between layers.

3) The influence of the error in the estimation of the radio channel on the power calculation. More accurate assessment is required.

4) The big computational complexity of processing the NOMA signal due to the fact you have to demodulate (and decode, if possible) all the lower-level channels to get data from the required high-level channel.

On the PD-NOMA system is affecting by various factors. For example, the influence of noise from a multipath radio wave channel, synchronization and equalization errors, etc.

This article discusses the influence of factors such as error estimation in channel, synchronization errors on the probability of a bit error when demodulating a PD-NOMA signal.

* Corresponding author: udzhon@mail.ru 


\section{Processing the PD-NOMA signal in the receiving device}

Consider a signal-processing algorithm using OFDM modulation and the PD-NOMA method. In the receiving device, a signal is digitizes. It represents the sum of the signals of two users.

OFDM symbol consisting of $N_{\mathrm{d}}$ samples written as:

$$
\begin{gathered}
\dot{s}(k)=\frac{1}{N_{\mathrm{d}}} \cdot \sum_{n=1}^{N_{\mathrm{d}}} \dot{x}(k) \cdot e^{i \cdot 2 \cdot \pi \cdot k \cdot n / N_{\mathrm{d}},} \\
k=\left[1: N_{\mathrm{d}}\right],
\end{gathered}
$$

where: $\dot{x}(k)$ is a complex modulated sequence. The OFDM symbol includes a cyclic prefix (CP) it size is $N_{\mathrm{g}}$. It regulates by the standard communication system.

Given a random time offset is not-multiple of the sampling interval, the mathematical representation of the OFDM symbol corresponds to the expression:

$$
\dot{s}(k)=\frac{1}{N_{\mathrm{d}}} \cdot \sum_{n=1}^{N_{\mathrm{d}}} \dot{x}(k) \cdot e^{i \cdot 2 \cdot \pi \cdot k \cdot\left(n / N_{\mathrm{d}}+\Delta \tau \cdot d f\right)}
$$

The sum signal of the two users written as:

$$
\dot{S}(k)=A_{1} \cdot \dot{s}_{1}(k)+A_{2} \cdot \dot{s}_{2}(k)
$$

where: $\dot{s}_{1}(k)$ is the signal for the first user, $\dot{s}_{2}(k)$ is the signal for the second user, $A_{1}$ is the amplitude multiplier of the first user, $A_{2}$ is the amplitude multiplier of the second user. These signals have different powers.

To demodulate a weaker signal, it is first necessary to demodulate and regenerate a more powerful signal. For this purpose, standard processing performed by including frame, frequency, and symbol synchronization. In addition, it including equalization and demodulation. Next, the signal is regenerated and subtracted from the received signal (SIC algorithm - successive interference cancellation). After that, a demodulation of a signal of lower power can be performed. Functional diagram of the signal processing algorithm is shown in Figure 1.

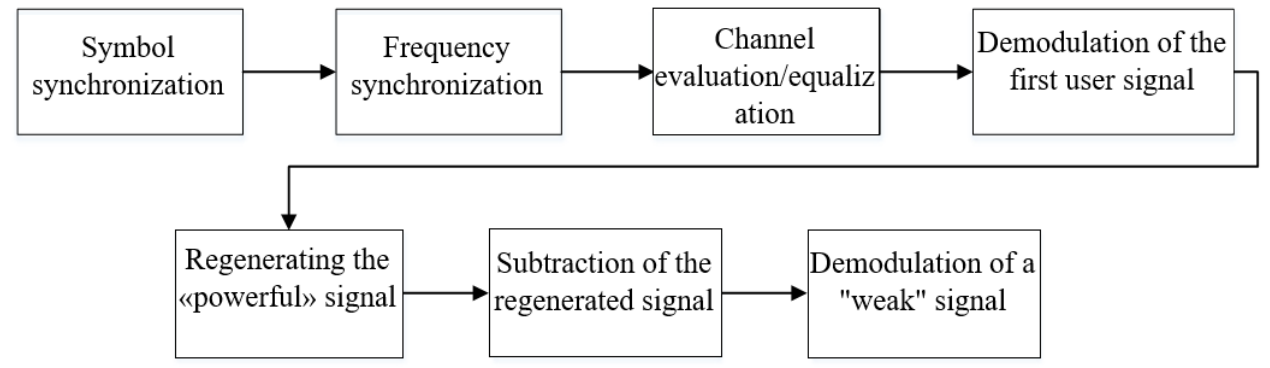

Fig. 1. PD-NOMA signal processing block diagram.

The main factors may affect demodulation are: 
- Symbol synchronization error,

- Frequency synchronization error,

- Error estimating transfer function of the communication channel.

Operation of the system can possible only with accurate frame synchronizations. If OFDM modulation is used, the symbol synchronization error removed by the equalizer and maybe not considered as a significant factor. However, the frequency synchronization error can have a significant influence. When using OFDM modulation, the frequency synchronization error leads to noise in the constellation and cannot corrected by the equalizer. The following section describes the influence of the factors listed on the probability of a bit error.

\section{Modeling of factors affecting the probability of a bit error of the PD-NOMA method}

To assess the influence of these factors on the operation of the communication system, using NOMA technology, mathematical modeling was perform in the Matlab environment. The following scenario of the system was considered during modeling.

The base station generates signals of two users with overlapping in the time-frequency domain. The SNR in the receiver of the first user is $20 \mathrm{~dB}$. The SNR in the receiver of the second user $30 \mathrm{~dB}$. Modulation for the first QPSK user. Modulation of the second QAM 16 user. The first user receives and demodulates his signal at the system interference. The second user receives and demodulates the first user signal. Then regenerates it and subtracts it from the received signal. Then demodulates its own signal.

This scenario illustrated in Figure 2.

Power distribution:

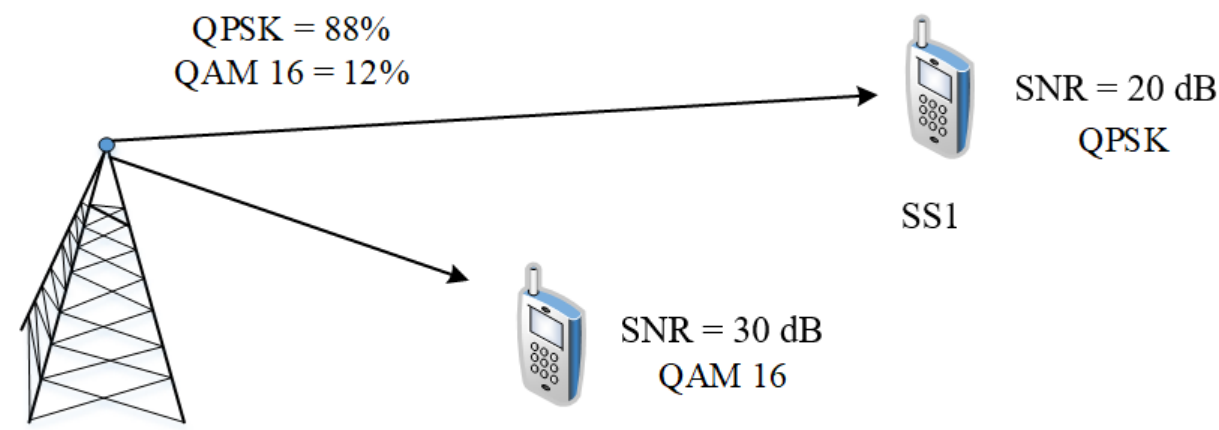

BS

SS2

Fig. 2. PD-NOMA system scenario.

Figure 2 indicates: SS1 is the first subscriber station, SS2 is the second subscriber station, BS is the base station. In the developed model is studied influence of the error in estimating transfer function of the radio channel. Also studied the error in estimating frequency shift on the probability of a bit error during signal demodulation.

\begin{tabular}{|l|l|l|l|l|l|l|}
\hline $\mathrm{CP}$ & Pilot signal & $\mathrm{CP}$ & Data 1 & $\mathrm{CP}$ & Data $\mathrm{K}$ \\
\hline
\end{tabular}

Fig. 3. Frame structure. 
The system parameters used in the simulation shown in the table:

Table 1. System parameters used in the simulation.

\begin{tabular}{|c|c|}
\hline Parameter & Value \\
\hline Number of users & 2 \\
\hline Modulation of the first user & QPSK \\
\hline Modulation of the second user & QAM 16 \\
\hline Power distribution QPSK & $88 \%$ \\
\hline Power distribution QAM16 & $12 \%$ \\
\hline The number of OFDM symbols, & 10 \\
\hline Signal band, MHz & 10 \\
\hline Sample rate, $f_{\mathrm{s}} \mathrm{MHz}$ & 10 \\
\hline Duration of the OFDM symbol without guard interval $\left(T_{b}\right), \mathrm{us}$ & 102,4 \\
\hline Duration of the cyclic prefix $(\mathrm{CP}) T_{g}=1 / 8 \cdot T_{b}, \mathrm{us}$ & 12,8 \\
\hline Subcarrier spacing, $\mathrm{kHz}$ & 9,765 \\
\hline Number of pilot subcarriers & 74 \\
\hline Modulation of pilot subcarriers & BPSK \\
\hline
\end{tabular}

The frame structure used in the simulation is shown in Figure 3.In the simulation multipath channel, parameters recommended by international telecommunication union were used. These parameters shown in table 2 [3].

Table 2. Parameters of the multipath channel used in the simulation.

\begin{tabular}{|c|c|c|}
\hline Pass number & Delay, ns & Attenuation, dB \\
\hline 1 & 0 & 0 \\
\hline 2 & 200 & $-0,9$ \\
\hline 3 & 800 & $-4,9$ \\
\hline 4 & 1200 & $-8,0$ \\
\hline 5 & 2300 & $-7,8$ \\
\hline 6 & 3700 & $-23,9$ \\
\hline
\end{tabular}

The error in estimating the channel parameters in the receiving device directly affects the operation of the sequential interference suppression (SIC) algorithm [4] and further signal demodulation.

Figure 4 shows the true and estimated transfer functions of the channel, the values of the normalized RMS error and the corresponding signal constellations. The normalized RMS error of the estimation of the transfer function determined by the expression [5]:

$$
\begin{gathered}
\text { NMSE }=\frac{1}{N} \sum_{i=1}^{N} \frac{\left(\hat{T F_{i}}-T F_{i}\right)^{2}}{\overline{\hat{T} F_{i}} \cdot \overline{T F}}, \\
\overline{\hat{T} F_{i}}=\frac{1}{N} \sum_{i=1}^{N} \hat{T} F_{i}, \\
\overline{T F_{i}}=\frac{1}{N} \sum_{i=1}^{N} T F_{i}
\end{gathered}
$$


where: $N$ is the number of counts of the transfer function, $\hat{T} F$ is the estimation of the transfer function, $T F$ is the true value of the transfer function, $i$ is the count number.

$$
N M S E=5 \cdot 10^{-4} \text {, }
$$
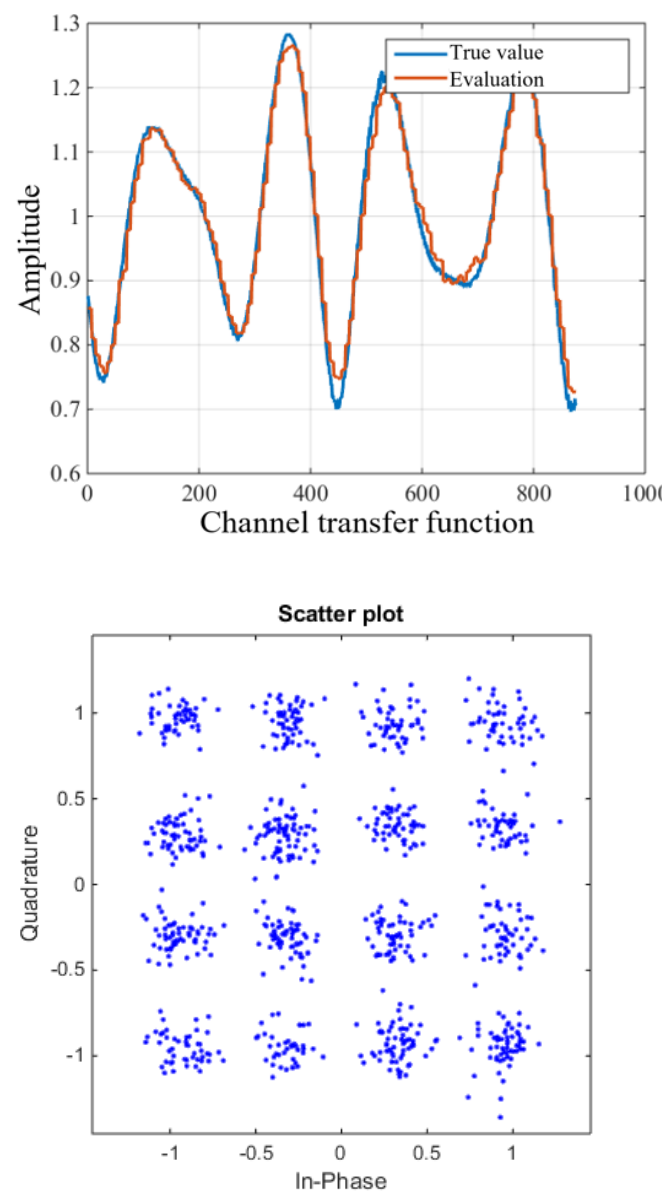

$N M S E=15 \cdot 10^{-3}$,
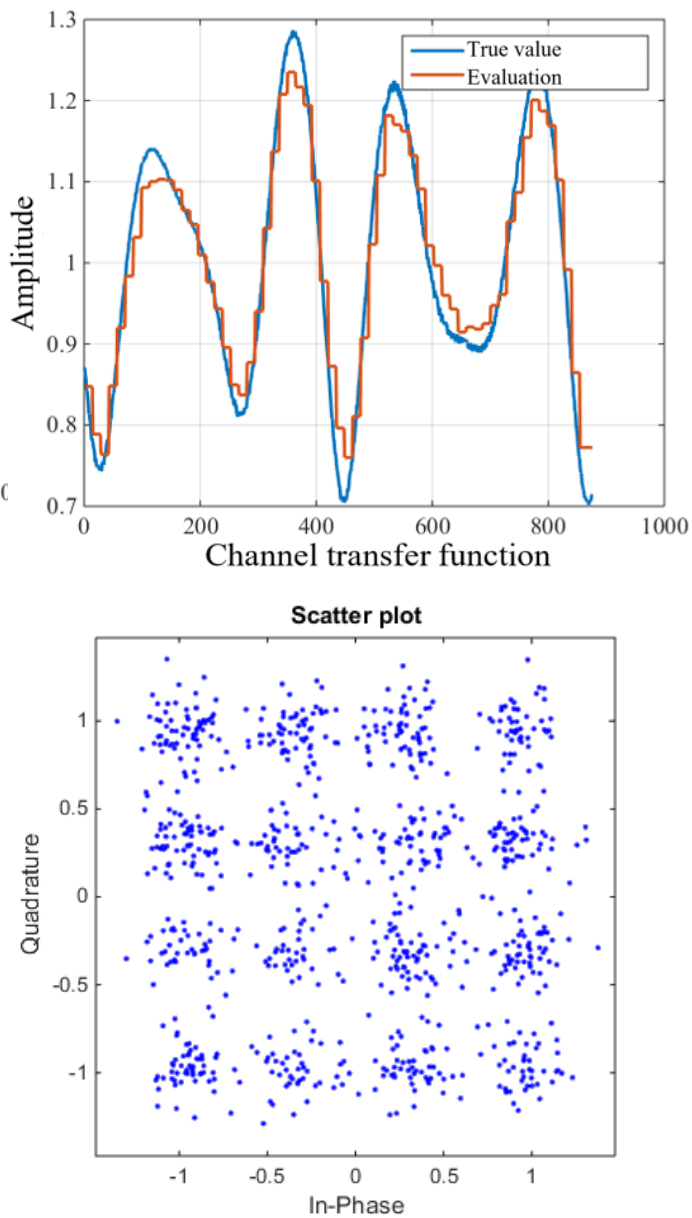

Fig. 4. Transfer function and signal constellations at different values of the normalized RMS error of the estimation of the transfer function.

Figure 5 shows the probability of a bit error of the normalized RMS error of the estimation of the transfer function.

Figure 5 shows the error in estimating the transfer function by the value of $N M S E=0.2 \cdot 10^{-3}$, leads to a significant change in the probability of a bit error (by an order of magnitude).

An important factor affecting the operation of the PD-NOMA method using OFDM modulation is synchronization errors in both the time and frequency domain. Errors in the time domain lead to phase distortions. It corrected by the equalizer [6]. It is known, when using OFDM modulation, the frequency offset have a different effect on the probability of an error in demodulation of the signal. It depends on the SNR, the signal band and the number of carriers used. 


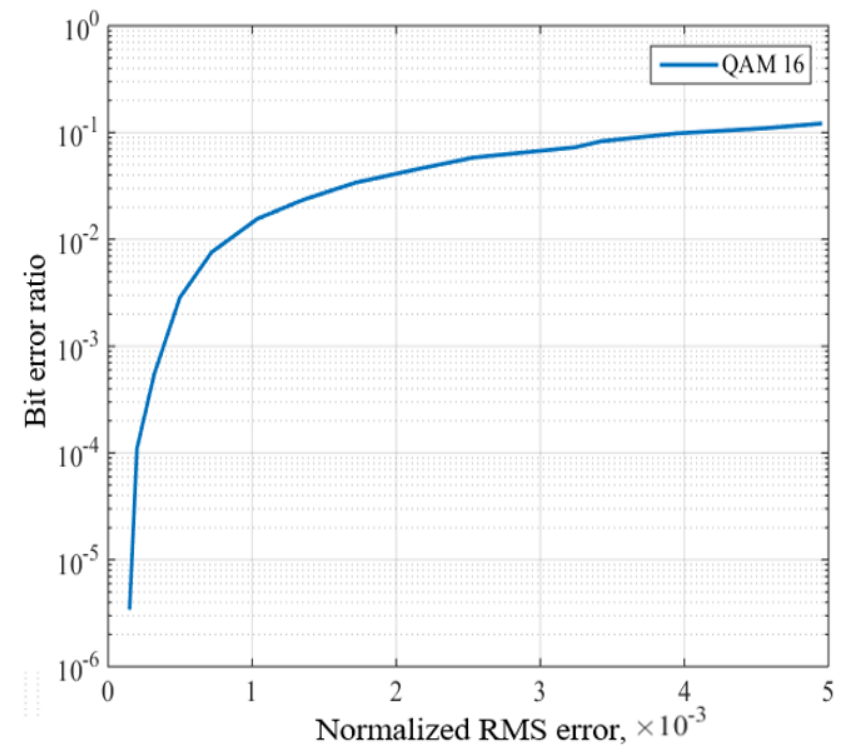

Fig. 5. Dependence of the probability of bit error on the normalized RMS error of the estimate of the transfer function.

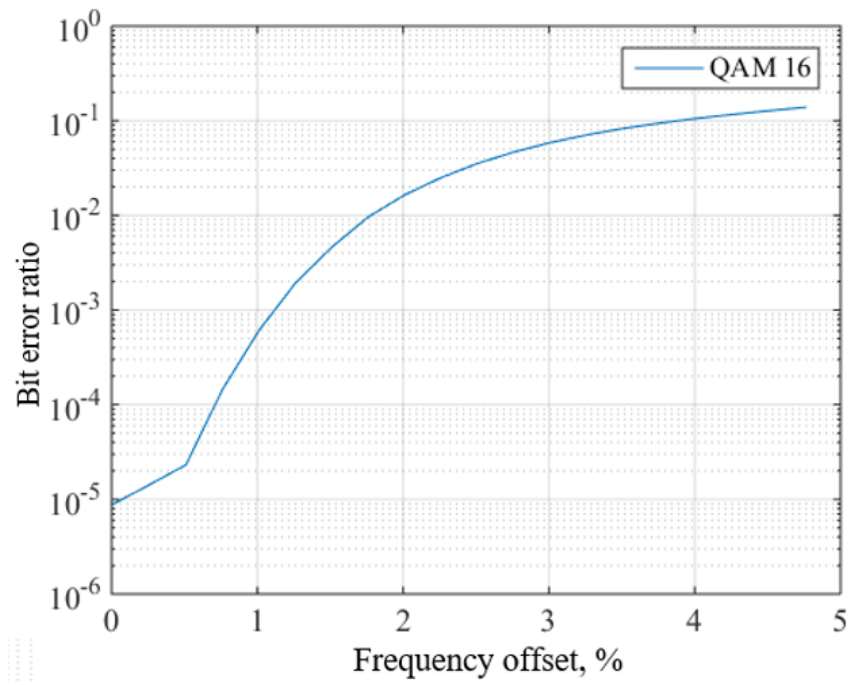

Fig. 6. Dependence of the probability of bit error on the ratio of the frequency shift error value to the frequency interval between subcarriers.

The closer the sub-carriers are located, the greater effect on the same frequency offset. Due it in this model, the dependence of the probability of a bit error on the ratio of the error value of the frequency offset to the frequency interval between the subcarriers was investigated.

Figure 6 shows the dependence of the probability of bit error on the ratio of the frequency shift error value to the frequency interval between subcarriers. Using the PD NOMA and OFDM modulation methods, even a small, about $1 \%$ of the frequency shift from the interval between subcarriers, leads to a significant increase in the probability of error. 
Also, the operation of the PD NOMA system affected by errors during demodulation of higher power subscribers. Each incorrectly demodulated symbol of one user, correspond a greater multiple of the number of incorrectly demodulated symbols of signals with lower power. Power level depends on the modulation index used in the system.

\section{Conclusion}

This article provides an overview and analysis of factors affecting the operation of a communication system using the PD NOMA method. The main factors affect the probability of a bit error during signal demodulation include a channel estimation error, signal/noise ratio, synchronization errors. The simulation performed for PD NOMA system using OFDM modulation, for fixed values of the SNR. However, with different values of the error of the estimation of the transfer function and error synchronization. An important factor affecting the probability of error during demodulation is the correct power distribution. Also important the absence of errors in demodulation of subscribers' signals with the higher power.

This work was funded by the Grant of the President of the Russian Federation, Project Number MK1126.2019.9.

\section{References}

1. Saito Y. et al. Non-orthogonal multiple access (NOMA) for cellular future radio access //2013 IEEE 77th vehicular technology conference (VTC Spring). - IEEE, pp. 1-5 (2013)

2. Kryukov Ya.V, Demidov A.Ya, Pokamestov D.A, Multiple access method with separation of channels by power on orthogonal carriers. T-CommTelecommunications and Transport. V. 12. №. 1 (2018)

3. Channel Models A Tutorial [Electronic resource]. - Access mode: http://www.cse.wustl.edu/ jain/cse574-08/ftp/channel_model_tutorial.pdf, free (access date 03.17.2019)

4. Usman M. R. et al. On the performance of perfect and imperfect, SIC in downlink non-orthogonal multiple access (NOMA) //2016 international conference on smart green technology in electrical and information systems (ICSGTEIS). - IEEE, p. 102106 (2016)

5. Bendat J, Piersol A, Applied analysis of random data: Wiley, 2010. 640 p. 4th ed.

6. The study of equalization methods for communication systems using OFDM signals / R.R. Abenov, A.S. Vershinin, E.P. Voroshilin, E.V. Rogozhnikov. Bulletin of SibGUTI, p. 50-56 (2013) 